\title{
PROGRESSION OF FUNGAL MICROBIOTA IN CYSTIC FIBROSIS PATIENTS
}

C de la Horra, L Carrasco-Hernández, V Friaza, R Morilla, I Delgado-Pecellín, J Medrano, E Quintana-Gallego, EJ Calderon.

Institute of Biomedicine Seville. Virgen del Rocio University Hospital. Ciber-ESP

\section{INTRODUCTION}

The presence of fungi in the airways of patients with cystic fibrosis (CF) is common; the most prevalent fungi described in these patients using conventional culture methods are Aspergillus and Candida. However, the presence of fungal microbiota is underestimated and it is unknown its role in the disease progression.

\section{OBJECTIVE}

Describe changes over the time of fungal microbiota and evaluate clinical parameters related to the Cystic Fibrosis patients

\section{METHODOLOGY}

Prospective observational cohort study including $60 \mathrm{CF}$

Clinical, functional data and sputum at 0 and 6 months were collected

ITS identification
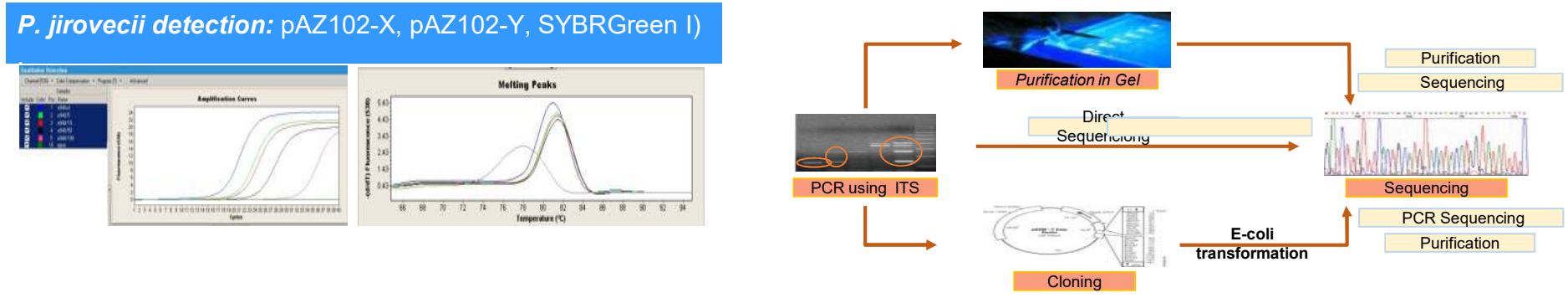

\section{RESULTS}

\begin{tabular}{|c|c|c|c|c|}
\hline & \multicolumn{2}{|l|}{0} & \multicolumn{2}{|l|}{6} \\
\hline Rate & 42 & & 39 & \\
\hline Isolates & 62 & & 62 & \\
\hline $\begin{array}{l}\text { Candida } \\
\text {-albicans } \\
\text {-Parapsilosis } \\
\text {-Glagrata } \\
\text {-Tropicallis } \\
\text {-Galli } \\
\text {-others }\end{array}$ & $\begin{array}{l}16.1 \\
9.6 \\
1.6 \\
- \\
- \\
1.6\end{array}$ & & $\begin{array}{l}20.9 \\
3.2 \\
6.4 \\
4.8 \\
- \\
1.6\end{array}$ & \\
\hline P. jirovecii & 29.1 & & 30.6 & \\
\hline $\begin{array}{l}\text { Aspergillus } \\
\text {-spp } \\
\text {-restrictus }\end{array}$ & $\begin{array}{l}11.2 \\
-\end{array}$ & & $\begin{array}{l}4.8 \\
1.6\end{array}$ & \\
\hline $\begin{array}{l}\text { Cladosporium } \\
\text {-sp } \\
\text {-cladosporoides } \\
\text {-herbatum } \\
\text {-ramoterellum }\end{array}$ & $\begin{array}{l}4.8 \\
1.6 \\
- \\
-\end{array}$ & & $\begin{array}{l}6.4 \\
- \\
1.6 \\
-\end{array}$ & \\
\hline $\begin{array}{l}\text { Criptococcus } \\
\text {-sp } \\
\text {-flavescens } \\
\text {-deformans }\end{array}$ & $\begin{array}{l}1.6 \\
1.6 \\
1.6\end{array}$ & & $\begin{array}{l}- \\
-\end{array}$ & \\
\hline $\begin{array}{l}\text { Uncultured fungi } \\
\text {-Lewia } \\
\text {-Davidella }\end{array}$ & $\begin{array}{l}1.6 \\
1.6\end{array}$ & & 3.2 & \\
\hline Ascomycetes & 1.6 & & - & \\
\hline Others & $\begin{array}{l}\text { Titricum } \\
\text { Dioscorea } \\
\text { Rhodosporium } \\
\text { G. Fragans }\end{array}$ & $\begin{array}{l}1.6 \\
3.2 \\
1.6 \\
1.6 \\
1.6\end{array}$ & $\begin{array}{l}\text { Sacharomyces } \\
\text { Alternaria } \\
\text { Wallemia sebi } \\
\text { Trichoporum } \\
\text { Sporobolomyces } \\
\text { Malasezia } \\
\text { Pheniophora } \\
\text { Zygosacharomyces }\end{array}$ & $\begin{array}{l}1.6 \\
3.2 \\
1.6 \\
1.6 \\
1.6 \\
1.6 \\
1.6 \\
1.6\end{array}$ \\
\hline
\end{tabular}

At $\mathrm{t}=0$ colonized $\mathrm{CF}$ patient presented a lower FEV1 higher proportion of inhaled steroids use. At 6 months: isolates were persistent in $47.6 \%$

$11.1 \%$ presented new isolates in negative subjects.

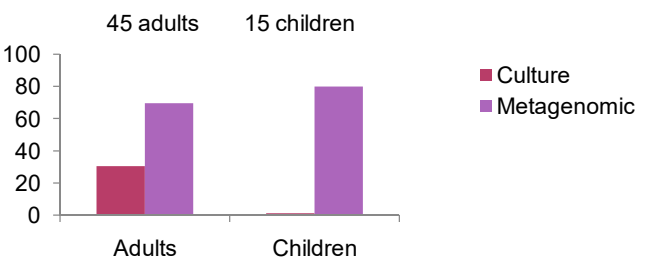

Mycobiota progression

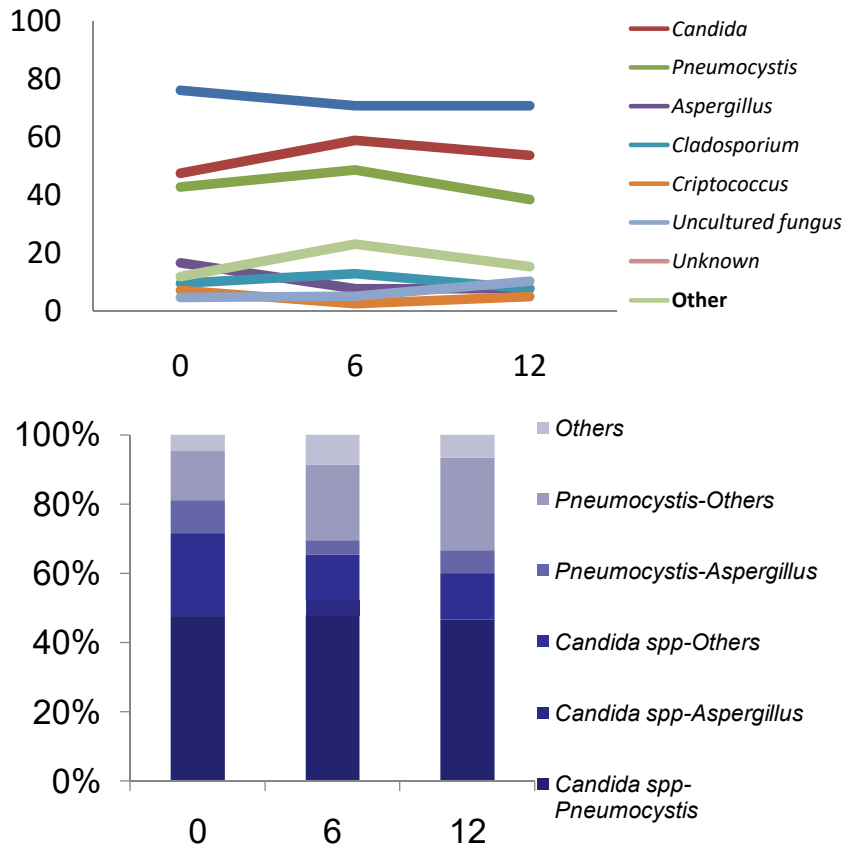

Patients with previous fungus isolation, presented a worse lung function FEV1 $(-120 \mathrm{~mL})$ as compared with persistent negative patients $(+109 \mathrm{~mL} ; \mathrm{p}=0.088)$

\section{CONCLUSION}

Fungi colonization in the airway measured by metagenomic techniques provides information on the prevalence and factors associated with this colonization. This technique enables the detection of unknown and uncultivable fungi especially in children and identifies a larger population of fungal microbiota in CF that remains over time.

Newly acquired isolations have an impact on the disease progression 